\title{
Pediatric Biobanking: Kids Are Not Just Little Adults
}

\author{
Edited by Daniel R. Catchpoole ${ }^{1}$ \\ Authors: David Carpentieri, ${ }^{2}$ Suzanne Vercauteren, ${ }^{3}$ Lalita Wadhwa, ${ }^{4}$ William Schleif, ${ }^{5}$ \\ Li Zhou, ${ }^{6}$ Junmei Zhou, ${ }^{7}$ Rania M. Labib, ${ }^{8}$ Elke Smits, ${ }^{9,10}$ and Engela H. Conradie ${ }^{11}$
}

\section{Editor's Introduction}

$\mathrm{P}$ EDIATRIC BIOBANKING BRINGS a unique set of challenges and complexities to an already multifaceted system. To address these specific issues, an ISBER Pediatric Special Interest Group (SIG) was formed in September 2017. This pediatric biobanking SIG focuses on the complex and unique aspects of biobanking as related to pediatric biobank participants and pediatric samples. This SIG consists of members from New Zealand, Australia, China, Egypt, Belgium, United States, Europe, South Africa, and Canada who manage biobanking activities that have a focus on facilitating pediatric research. Many of these biobanks operate as embedded facilities within children's hospitals that presents a biobanking environment that requires management of biospecimens from a highly defined and vulnerable patient population.

\section{We asked leading pediatric biobanks from across the world to tell us what makes pediatric biobanking special?}

Their responses highlight how all pediatric biobanks engage vulnerable populations of patients and their parents/ families into research, and that these patients represent many rare disease scenarios. As pediatric diseases are often considered rare, pediatric biobanks must operate with a long-term viewpoint. Pediatric biobanking is an important facilitator of research into childhood disease but it has its unique challenges in that the participants are minors and considered a vulnerable population. Consent to donate specimens and clinical data to biobanks involves third-party approval being obtained from parents or legal guardians with assent of children. The age at which assent is obtained varies by pediatric biobank, and some places require written whereas others only verbal assent. Practices differ across jurisdictions and thus decisions about these matters become an institutional ethics board led policy. Working with children presents added ethical barriers due to vulnerability that need to be considered, often necessitating a more dynamic consenting environment. As biobanks have the capability of storing specimens and clinical data for many years, reconsent of participants at the age of majority is a contentious and potentially expensive issue for pediatric biobanks. In addition, a child may be deemed to have the "capacity to consent" but must be able to show a full understanding of the process they are participating in. If this capacity is acceptable, then the need for parental consent is no longer needed. In sum, clearly consenting participants to a pediatric biobank involves more than consenting to an adult biobank.

Pediatric biobanks struggle to be reactive to specific researcher needs, but work best as hospital embedded practices, operating close to the source of the tissue distribution found in pathology departments, following standardized tissue handling steps over the long term. Pediatric biobanks need to capture biospecimens early in a patient's clinical journey as many disorders they face have a congenital basis that does not become clinically meaningful until later in life. Early intervention and investigation are often needed where routine tissue collection supporting meaningful research is identified at the clinical interface. As the needs of pediatric researchers become increasingly specific, pediatric biobanks are required ever more than their adult counterparts to work within networks with prospective biobanking opportunities facilitating multicenter research either through clinical trials or as core translational research efforts. The contributions mentioned highlight how pediatric biobanks around

${ }^{1}$ Children's Cancer Research Unit, Kids Research, The Children's Hospital at Westmead, Westmead, Australia.

${ }^{2}$ Pathology, Phoenix Children's Hospital, Phoenix, Arizona, USA.

${ }^{3}$ Haematology, British Columbia Children's Hospital, Vancouver, Canada.

${ }^{4}$ Divisions of Congenital Heart Surgery, Texas Children's Hospital, Houston, Texas, USA.

${ }^{5}$ Pediatric Biorepository, Johns Hopkins All Children's Pediatric Biorepository, St. Petersburg, Florida, USA

${ }^{6}$ Tumour Bank, Children's Cancer Research Unit, Kids Research, The Children's Hospital at Westmead, Westmead, Australia.

${ }^{7}$ Central Laboratory and Biobank, Shanghai Children's Hospital, Shanghai, China.

${ }^{8}$ Biorepository and Biospecimen Research, Children's Cancer Hospital, Cairo, Egypt.

${ }^{9}$ EPTRI Belgian JRU, Belgium.

${ }^{10}$ Clinical Research Center, University of Antwerp/Antwerp University Hospital, Edegem, Belgium.

${ }^{11}$ North-West University, Potchefstroom Campus, Centre for Human Metabolomics, Potchefstroom, South Africa. 
the world are adjusting to these research needs, and creating their own unique contributions to our biomedical communities.

Address correspondence to: Daniel R. Catchpoole, PhD Head, Tumour Bank Children's Cancer Research Unit Kids Research The Children's Hospital at Westmead Locked Bag 4001 Westmead, NSW 2145 Australia

E-mail: daniel.catchpoole@health.nsw.gov.au

\section{Expert Response: David Carpentieri, Phoenix Children's Hospital Biorepository, Arizona, USA}

"Nature is nowhere accustomed more openly to display her
secret mysteries than in cases where she shows traces of her
workings apart from the beaten path; nor is there any better
way to advance the proper practice of medicine than to give
our minds to the discovery of the usual law of nature by the
careful investigation of cases of rarer forms of disease. For it
has been found in almost all things, that what they contain of
useful or of applicable nature, is hardly perceived unless we
are deprived of them, or they become deranged in some way."
Dr. William Harvey, 1657

$\mathrm{D}$ R. HARVEY'S STATEMENT STILL RINGS true as the activities at the Phoenix Children's Hospital and Biorepository $(\mathrm{PCHB})$ support the research needed to better understand and care for the children we advocate for. As such, the PCHB is a division of the hospital's pathology department, serving as a liaison between our patients and the specialists requiring processing, storage, and tracking of well-preserved samples for clinical and research needs. More importantly, in the context of limited samples, the PCHB is responsible for supporting the research needs while maintaining adequate care of an extremely vulnerable population. To achieve this goal, the PCHB standard operating procedures for phlebotomy as well as for the collection of leftover samples are maintained through the main laboratory electronic system. In this capacity, the PCHB can sustain standards across the operations that led to our accreditation by the College of American Pathologists (CAP), the third pediatric center in the country and the first in the southwest region to receive the designation.

The uniqueness of our service extends beyond the diseases we support. Our customizable Health Insurance Portability and Accountability Act compliant electronic tracking system allows for the capture of valuable pediatric data such as age (hours, days, and months), history of prematurity, and diagnostic codes (ICD-9 and ICD-10) specific for this group.

Currently, the PCHB holds thousands of clinical and research specimens preserved at room air, frozen at several temperatures including liquid nitrogen vapor, and formalinfixed paraffin embedded tissues. Leftover clinical samples become available for open research when patients are enrolled in the Biological Material Availability Program (BMAP). The consent allows for the retrospective and prospective utilization of these clinical samples until the age of 18 years. Re- consenting at this age is now limited to samples requested for new research projects only. This approach derived from our historical low return when trying to capture all participants.

BMAP is a multidisciplinary program driven by the divisions interested in future research projects. A video ${ }^{1}$ explains the biobank concept and facilitates the enrollment of patients by their coordinators who are trained by the PCHB. The program is now expanding to a variety of rare disease clinics-lysosomal storage diseases, disorders of sexual development, etc. - and patients are added to the research inventory on an exponential basis.

As a member of the research team, we also oversee new projects at inception to better accommodate the research needs without compromising clinical care. Overall, the utilization of research specimens, BMAP and other projects, is overseen by the PCH Office of Research with the Biorepository Governance Committee, a group that includes our administration and medical specialists. These individuals are responsible for regulatory compliance and for the oversight utilization of our resources while protecting our patients' ethical and legal rights.

Currently, the PCHB not only supports a variety of clinical laboratory validation projects but also provides samples for neuroscience and oncology research. Among the many projects, the cerebrospinal fluid collection has provided invaluable samples to several national collaborative biomarker discovery studies and saliva is being utilized for an international cerebral palsy genomic study. Our quality efforts to preserve the most of small leftover samples are best exemplified by our NIH-supported dry plasma spot project for downstream proteomic applications.

Finally, the importance of our combined clinical and research operations to the quality of care and research at our institution has led to the invaluable recognition by our hospital administration. As such, this allows for our program to advocate and dream about improving the care of children who often harbor rare and neglected diseases.

\section{Reference}

1. PCH Biorepository, English version. YouTube video, 2:19. October 12, 2018. https://www.youtube.com/watch?v= WE5I8DP6SNs

Address correspondence to: David Carpentieri, $M D$ Pathology

Phoenix Children's Hospital 1919 E. Thomas Road Phoenix, AZ 85016

USA

E-mail: dcarpentieri@phoenixchildrens.com; pchbiorepository@phoenixchildrens.com

\section{Expert Response: Suzanne Vercauteren, British Columbia Children's Hospital BioBank, Canada}

$I_{p}^{\mathrm{N}}$ NNOVATION IN DIAGNOSTICS AND HEALTH treatment practices is only made possible through cutting-edge research. This is especially important for underserved populations such as rare disease patients and those with chronic complex disease. Having high-quality specimens readily available for approved research through the BC Children's 
Hospital BioBank (BCCHB) helps to fuel important discoveries for patients and improves time to discovery.

BCCHB, established in 2015, is the first pediatric and maternal/neonatal institutional biobank in Canada. The BCCHB routinely collects biospecimens with annotated data from individuals with a wide range of diseases and conditions as well as from healthy individuals seeking care at BC Children's Hospital $(\mathrm{BCCH}), \mathrm{BC}$ Women's Hospital, and BC Children's Healthy Minds Centre, allowing collections to be ready when the research question presents itself. The pediatric patient population is often underrepresented in medical research due to ethical issues and difficulty of sample collections. Benefits of a hospitalwide universal biobank for children not only include the benefits of standardized processes such as quality and consistency in consenting, specimens, and data, but also the opportunity to provide control specimens (as healthy children are rarely approached for control specimens), and the ongoing collections of often rare diseases, to name a few. The BCCHB has established processes that enable specimens to be used to their fullest potential — an important consideration in pediatric biobanking. The use of specimens leftover from clinical testing and processing a single specimen into multiple aliquots, so that one specimen can contribute to numerous studies, are processes to use every sample from a child to the fullest.

The BCCHB has facilitated major local and national research projects. At the request of families, autopsies were performed for biobanking purposes only, as per the wish of patients to have tissue removed to allow important research. Bereaved parents have also requested biobanking after stillbirths.

For biobanks to be successful, patient and public involvement and engagement are essential. The BCCHB has ensured that the patient voice is being heard in our processes. We have surveyed pediatric patients and parents, healthy adolescents and their parents, as well as parents from mental health units to learn of the opinions and concerns around pediatric biobanking. These engagement efforts have resulted in simplifying the consent process among other improvements. At $\mathrm{BCCH}$, we have a verbal permission process wherein families in distress are asked for permission to obtain and store diagnostic specimens followed by full consent at a later date. We have developed an electronic consent method to simplify the consent process and increase engagement.

Address correspondence to: Suzanne Vercauteren, PhD, MD, FRCPC Haematology British Columbia Children's Hospital 4500 Oak Street Vancouver, $B C$ V6H 3N1

Canada

E-mail: svercauteren2@cw.bc.ca

\section{Expert Response: Lalita Wadhwa, Texas Children's Hospital, Texas, USA}

$\mathrm{D}$ ISEASE-FOCUSED BIOBANKS of several pediatric disorders for purposes of optimizing treatment for patients with a particular common condition and tracking outcomes are very few. Pediatric disorders have unique characteristics and pro- gression pathways that require deeper investigations and focused research. The constantly changing developmental physiology of children involves multiple molecular and cellular pathways. With the possibility of an alteration in one pathway affecting multiple pathways, children are at increased risk to develop more complex syndromes than adults. In parallel to deducing different genes that play a role in disease development, the understanding of gene-environment interactions in children is of utmost importance. Several pediatric disorders have associated comorbidities that develop later in life, thus early intervention is necessary. Longitudinal and systematic collection of data and biospecimens starting from the neonatal period enables longterm research to examine genetic, epigenetic, and epidemiological influences on pediatric disease.

Congenital heart disease (CHD) is one such pediatric condition and represents the most common type of birth defect, with an estimated prevalence of 4 to 50 per 1000 live births. CHD affects $\sim 36,000$ children each year in the United States. The prognosis of children with complicated and uncomplicated CHD continues to improve with advancing surgical techniques and medical management; however, postoperative complications and long-term morbidities including neurodevelopmental problems continue to remain a challenge. Both genetic and environmental factors are known to be associated with $\mathrm{CHD}$, and yet the etiology of CHD remains complex and poorly understood. Epidemiologic studies have shown increased CHD recurrence risk in future pregnancies and a growing population of adults with CHD. Despite advances in technology such as next-generation sequencing, a better understanding of mechanisms of CHD development is still lacking.

Disease architecture of CHD differs widely in children and adults. The childhood disease has more heterogeneity in both disease phenotype and etiology and is often associated with a worse prognosis. Moreover, therapeutic and preventive measures in ischemic heart failure in adults have often been extrapolated in pediatric patients with CHD without extensive and focused pediatric research. The molecular mechanisms related to impact of cardiopulmonary bypass and reperfusion injuries on neonatal myocardium have not been elucidated. Data on the pathological progression from fetal to neonatal stages are also scarce. All of these factors despite surgical corrections are associated with higher mortality in the pediatric group in contrast to older age groups.

Overcoming these challenges needs to be enabled by a standardized collection of myocardial tissues within a biobank. An improved understanding of underlying molecular signature warrants an embedded system of longitudinal collection of these samples from neonatal stages to adulthood of CHD patients.

The Heart Center Tissue Bank (HCTB) at Texas Children's Hospital, established in 2010, is one of largest disease-focused tissue banks in the United States for CHD. HCTB currently houses $>10,000$ samples from pediatric patients with CHD. Samples range from waste heart tissue resected from surgical repairs, heart transplants and ventricular device placements, matched blood, and urine samples. Rare normal and diseased heart tissue specimens and matched blood samples supported with longitudinal clinical data sets make HCTB a highly unique resource of heart specimens with an ability to perform in-depth research of tissue-specific postzygotic molecular changes.

One research study that recently received $\$ 2.2$ million federal funding is to study the dynamic processes and cellular players linked to discrete subaortic stenosis, a rare 
form of CHD in children born with an obstruction in heart flow leading to significant mortality due to recurrence of obstruction after surgery. HCTB is the only resource in the world with large standardized and longitudinal collection of rare subaortic membrane tissue specimens from DSS patients and this project also highlighted a synergistic relationship between multidisciplinary fields of tissue banking, surgery, and bioengineering that has enabled understanding the underlying causes of this rare and complex pediatric heart disease.

Significant interindividual variability is known to exist in outcomes among patients with CHD. Not all patients with the same diagnosis have the same prognosis, response to surgery, response to medications, or outcomes. Reports also indicate predominance of certain defects in specific ethnic groups. Establishing large cohorts from an ethnically diverse pediatric population with a wide range of congenital defects along with relevant clinical data makes HCTB an ideal and the largest resource for all types of cardiac research including target discovery and validation, developing personalized tissue-engineered therapies that are compatible with developing bodies of infants, identifying underlying pediatric-specific genetic factors, and biomarker discovery for prevention or early detection of CHDs.

Address correspondence to: Lalita Wadhwa, PhD Divisions of Congenital Heart Surgery Texas Children's Hospital 6621 Fannin Street, Legacy Tower, 19th Floor Houston, TX 77030

USA

E-mail: 1xwadhwa@texaschildrens.org

\section{Expert Response: William Schleif, Johns Hopkins All Children's Hospital, Florida, USA}

$\mathrm{E}$ STABLISHED IN 2013 at a time when few pediatric biobanking models existed, the Johns Hopkins All Children's (JHAC) Pediatric Biorepository was conceived in part with a premise, adapted from the precision medicine and quality/patient safety eras of the "right samples for the right study." Access to high-quality biospecimens with corresponding and comprehensive phenotypic data is a major bottleneck for research and requires development in both biobanking infrastructure and the biospecimen sciences driving sample management strategies. Margins are even more critical in pediatrics-both financial and scientific, wherein supporting finances are constrained as well as what is physically available to collect for research, and "mulligans" (i.e., a second chance) are not an option. With this in mind, the JHAC Pediatric Biorepository focuses on prospective cohort collections supporting investigator-initiated studies with institutional review board approvals representing all fields and subspecialties within pediatrics and pediatric surgery under a comprehensive quality management system accredited for biorepositories by the CAP. The CAP accreditation is important, although it is not specific to pediatric biorepositories, because it does attempt to match external inspectors with significant pediatric experience and relevance during the peer-to-peer physical inspection process. This step regularly reoccurs in the accreditation lifecycle, arguably as one of the most important, as it eclipses the value of standards by incorporating feedback that is more granular and personalized from recognized experts in the field. The CAP providence includes clinical trial sample support for industry-sponsored research and other consortiadriven protocols. Individual research studies each have their own consent sets, which, depending on study aims, may be broad but often tiered and specific to certain uses. Some but not all studies create sample collections conducive to future research, in which a specimen access committee comprising scientists, physicians, bioethicists, and community representatives determines access and provides governance.

Phase I development of the biorepository included integration with hospital clinical infrastructure, with operations independently overseen as a distinct department providing an interdisciplinary model of leadership able to respond to emerging scientific needs in research. This phase established the operating procedures and quality management aspects of single site and multi-institutional fluid-basedblood, saliva, urine, cerebrospinal fluid, etc.-sample collections, as well as nucleic acid isolation and characterization. This effort is a vital component in the Kids-Duration of Therapy for Thrombosis trial, a randomized controlled clinical trial investigating the duration of anticoagulation in pediatric provoked venous thromboembolism, which required a multiomic approach to fuel diagnostic and prognostic biomarker discovery and validation. ${ }^{1,2}$ Phase II aspects involved the development of proprietary tissue-based collection and management strategies - specifically evolving the cross-referencing of diagnostic pathology informatics and fluid-based derivative samples. The current Phase III stage expanded biorepository infrastructure into a dedicated research and education building providing a larger and more scalable footprint for higher complexity processing needs and an enhanced capability for larger studies. This effort coincides with parallel developments in JHAC clinical coordinating center capabilities designed to expand the scope of services required for pediatric multicenter studies. $^{3,4}$ This multifaceted approach inherently adds to the ability of the JHAC biorepository to offer a not-for-profit biobank alternative, dedicated to improving the health of children, to other hospitals and academic institutions interested in sample-driven research. Biospecimen science has driven all of these phases-wherein best practices are adopted when available or defined by needs of highly specialized downstream testing platforms. If these are not available for new sampling methodologies, the JHAC pediatric biorepository develops and implements collaborative new techniques with key indicators of quality assessed for service-based adoptability and shares these methods externally for wider peer review and public use.

\section{Acknowledgments}

The author gratefully acknowledges Neil Goldenberg, $\mathrm{MD}, \mathrm{PhD}$, associate dean for research, Johns Hopkins All Children's Hospital, and Hector L. Monforte, MD, biorepository medical director.

\section{References}

1. Goldenberg NA, Abshire T, Blatchford PJ, et al. Multicenter randomized controlled trial on Duration of Therapy for Thrombosis in Children and Young Adults (the Kids-DOTT trial): pilot/feasibility phase findings. J Thromb Haemost 2015;13:1597-1605.

2. Goldenberg NA, Everett AD, Graham D, Bernard TJ, Nowak-Göttl U. Proteomic and other mass spectrometry 
based "omics" biomarker discovery and validation in pediatric venous thromboembolism and arterial ischemic stroke: Current state, unmet needs, and future directions. Proteomics Clin Appl 2014;8:828-836.

3. Goldenberg NA, Daniels SR, Mourani PM, et al. Enhanced infrastructure for optimizing the design and execution of clinical trials and longitudinal cohort studies in the era of precision medicine. J Pediatr 2016;171:300-306.

4. Schleif WS, Goldenberg NA, Catchpoole DR. The "federated pediatric biocloud" model: State of the art and future prospects in pediatric biospecimen science. J Pediatr 2020; 221:S43-S48.

Address correspondence to: William Schleif, MS, MT Pediatric Biorepository JHAC Pediatric Biorepository 501 6th Avenue South St. Petersburg, FL 33701

USA

E-mail: billy.schleif@jhmi.edu

\section{Expert Response: Li Zhou, Tumour Bank, The Children's Hospital at Westmead, NSW, Australia}

B IOBANKS HAVE EMERGED as key resources of translational research. The importance of pediatric biobanks is more prominent because accrual of pediatric samples requires a long-term strategy due to the rarity of childhood diseases, small specimen volumes, and limited population of children. Despite this obvious value in pediatric biobanking, sustainability remains a major obstacle for most pediatric biobanks. Therefore, it is critical to identify a sustainable model for pediatric biobanking that continuously supports and accelerates advancements in pediatric translational research.

A single institutional biobank embedded in hospital clinical pathways is one of the most economic and efficient models for biobanking. It is especially advantageous for pediatric biobanks since its operational proximity to tissue source reduces costs, minimizes reliance of uncertain funding streams, and ensures efficiency of practice. Financial efficiency is vital for pediatric biobanks' sustainability, as longer sample accrual time results in higher running cost per sample and lower utilization rate in a relative short period of time for pediatric biobanks. Also, there are fewer funding opportunities for them.

The Tumour Bank at The Children's Hospital at Westmead (TB-CHW) is a successful example of a single institutional biobank embedded in hospital clinical pathways. It coordinates the collection, storage, and distribution of pediatric biospecimens donated by patients and obtained through the normal patient management pathways, which are made available to researchers from around the world through an application process.

Within the past 20 years, the TB-CHW has supported 109 projects both in Australia and around the world. There are 80 publications generated from research projects we have supported. Worthy to note, the average collection period for samples that TB-CHW provided to projects is $138 \pm 69$ months or over a decade. At the extremes, $27 \%$ of projects received samples collected over a period of 15 years or more. In addition, $>60 \%$ of the samples provided were studied alongside specimens collected from multiple sites. For $11 \%$ of the projects supported, the TB-CHW contribution to those studies was $<10 \%$ of the total collection that reflects the investigators undertaking a world-wide sweep for a particular very rare tumor. Apart from the academic outcomes, TBCHW has facilitated $>30$ clinical trial studies. Consequently, TB-CHW has gained its formal recognition from NSW Health Pathology as the first certified biobank in Australia.

Overall, TB-CHW's experience demonstrates the success of a sustainable pediatric biobank model - a single institution biorepository embedded in a hospital that is small in size, simple in structure, but mighty with regard to its contribution to academic outcomes and the meaningful impact of the research.

Address correspondence to: Li Zhou, PhD Tumour Bank Children's Cancer Research Unit Kids Research The Children's Hospital at Westmead Locked Bag 4001 Westmead, NSW 2145 Australia

E-mail: li.zhou@health.nsw.gov.au

\section{Expert Response: Junmei Zhou, Shanghai Children's Hospital, China}

$\mathrm{T}$ HERE IS A GOOD VARIETY of pediatric disease resources in Shanghai Children's Hospital. A standard biobank is needed to collect, handle, and preserve the biospecimen as well as integrate the corresponding clinical information, thus providing scientific management and biospecimen sharing. Our biobank was constructed step by step from 2012 with the support of findings from Shanghai Jiaotong University School of Medicine and Technology Commission of Shanghai Municipality. Up to now, an expert committee and an ethics committee for the operation of the biobank have been established; a professional management team including staff with dual backgrounds of clinical medicine and basic biology has been organized; a standard operating procedures system has been completed that could provide a guarantee for the concrete implementation of the biobank. The biobank is embedded in the research platform of a central laboratory that is composed of cell biology, molecular biology, and morphological platforms. For these reasons, our biobank is not only a service provider, but also a research center.

The biobank is currently focusing on the biospecimens of genetic and metabolic diseases, birth defects, tumors, etc. Biospecimens from $>30$ categories of pediatric diseases have been preserved in the biobank, including whole blood, serum, tissue blocks, DNA, and urine. All of these biospecimens, most of which are biomolecular-based and unregenerable, have supported several funding applications and scientific publications. However, with the development of biology and medicine, there is a growing interest in a living biobank in addition to the traditional biomolecular-based biobank. Living biobanks play an even more important role in illustrating mechanisms of congenital birth defects and metabolic diseases that are common in children's hospital. 
Owing to the advancements in cryopreservation technologies, a living biobank can preserve viable cells, tissues, organoids, and even whole organs for a long time. ${ }^{1}$ Several cell banks preserving both somatic cells and pluripotent stem cells have been established including stem cell banks, immune cell banks, and conditional reprogramming cell banks. ${ }^{2}$ Disease-specific induced pluripotent stem (iPS) cells have also been considered as an important research model for investigating congenital malformation, including cryptorchidism that is common in the department of pediatric urology. ${ }^{3}$ Meanwhile, organoids have been developed from either microtissue blocks or stem cells and served as valuable research models of diseases covering liver, pancreas, prostate, brain, etc. ${ }^{4}$ Both cells and organoids, as well as other forms of living models, have been applied in tremendous research. Through the development of a living biobank, we could consciously manage the living research models scattered in various research groups in a scientific way, which will help precious living models to be shared in a wider range and help translational medical research.

A typical application example in our living biobank is the cryptorchid-specific iPS cells that were established from urine-derived cells through introduction of key transcription factors. ${ }^{3}$ Urine was collected from a child with congenital cryptorchidism that will probably develop infertility in his adulthood. The in vitro germ cell differentiation of cryptorchid-specific iPS cells can be applied to investigate how infertility occurred and the possible mechanism. Other congenital and metabolic diseases that are common in pediatrics can also be investigated based on a strategically constructed living biobank. Our biobank is also exploring to establish organoids as another kind of living research resource. ${ }^{4}$ We hope to cooperate on establishing a robust living biobank on important and special pediatric diseases.

\section{Funding}

This study was supported by the Shanghai Municipal Commission of Health and Family Planning (Grant No. 201940204) and the Shanghai Collaborative Innovation Center for Translational Medicine (Grant No. TM201827).

\section{References}

1. Giwa S, Lewis JK, Alvarez L, et al. The promise of organ and tissue preservation to transform medicine. Nat Biotechnol 2017;35:530-542.

2. Brown KS, Rao MS, Brown HL. The future state of newborn stem cell banking. J Clin Med 2019;8:pii:E117.

3. Zhou JM, Wang X, Zhang SL, et al. Generation and characterization of human cryptorchidspecific induced pluripotent stem cells from urine. Stem Cells Dev 2013;22:717-725.

4. Li S, Wang M, Zhou JM. Brain organoids: A promising living biobank resource for neuroscience research. Biopreserv Biobank 2020;18:136-143.

Address correspondence to: Junmei Zhou, MD, PhD

Central Laboratory and Biobank Shanghai Children's Hospital 1400 Beijing Road West Shanghai 200040

China

E-mail: junmei_zhou@139.com

\section{Expert Response: Rania M. Labib, Children's Cancer Hospital (CCHE)- 57357-Biorepository, Egypt}

$\mathrm{C}^{\mathrm{n}}$ HILDREN's CANCER HOSPITAL (CCHE)-57357-Biorepository and Biospecimen Research Facility collects, processes, and stores samples from pediatric patients who have cancer and their parents for research purposes. All samples are annotated with all the epidemiological and clinical data through Cerner $^{\circledR}$ : our electronic health care system. CCHE Biorepository stores $>40,000$ human samples of a variety of pediatric cancer types and $>20,000$ pathogen strains.

I have been leading the CCHE Biorepository since its establishment in 2009 with the vision to foster research and promote cures through integrating research into all our services as a centralized core facility that provides researchers with samples linked with clinical, epidemiological data, and biospecimen-related data. In November 2012, we started sample collection including blood, tissue, and cerebrospinal fluid (CSF) from pediatric cancer patients at different clinical time points (initial diagnosis, relapse, and remission). Then, we started a general collection of a blood samples from both of the child's parents that will allow for triogenetic studies. These parental samples will allow us to provide support for researchers who want to study the genetic epidemiology of pediatric cancers and we thought that this will be helpful as we do not provide control age-matching samples. In 2014, we started a section for pathogen biobanking, coordinating with the microbiology central laboratory at $\mathrm{CCHE}$ where we store different strains of pathogen and resistant strains. ${ }^{1}$

We have a very good collection success rate and participants were very cooperative and willing to donate their samples for research. ${ }^{2}$ Therefore, starting in 2018, the CCHE Biorepository has started sample release and provided samples to different research projects. We not only provide the retrospective collection of samples, but we also sometimes help researchers to collect prospective specific sample types with specifics in collection and/or timing. We continually do quality control on all our steps as well as on sample quality. The release of samples is in coordination with the scientific medical and advisory committee and biorepository coordinating committee at CCHE-57357.

We are members in ISBER, ESBB, and BCNet. Also, the $\mathrm{CCHE}$ Biorepository provides trainings, site visits, and consultations to help establish different biobanks in Egypt and Africa.

\section{References}

1. Labib RM, Mostafa MM, Alfaar AS, et al. Biorepository for pediatric cancer with minimal resources: Meeting the challenges. Biopreserv Biobank 2016;9:16.

2. Labib RM, Hassanain O, Alaa M, et al. Planning today for tomorrow's research: Analysis of factors influencing participation in a pediatric cancer research biorepository. Front Oncol 2017;7:324.

Address correspondence to: Rania M. Labib, PhD, MPharm, BPharm Biorepository and Biospecimen Research Children's Cancer Hospital Cairo 57357

Egypt

E-mail: rania.labib@57357.org; bcc@57357.org 


\section{Expert Response: Elke Smits on Behalf of the European Paediatric Translational Research Infrastructure Belgian Joint Research Unit, Belgium}

$\mathrm{T}$ HE BELgIAN JOINT RESEARCH UNIT (JRU) for participation as a national node in the European Paediatric Translational Research Infrastructure (EPTRI) is in the preparatory phase. ${ }^{1}$ EPTRI is a new distributed research infrastructure aimed to create a framework for basic and translational pediatric research to facilitate the development of new and innovative medicines specifically designed to address the needs of babies, children, and adolescents so that they will have access to them. ${ }^{2}$ The operational activity will be based on a hub and spoke model. In addition to the coordination and networking activities, the central HUB will also act to bridge EPTRI and the pediatric initiatives focused on clinical steps to make the whole pediatric drug development process more efficient and strategic. Information technology and tools will be used intensively to maintain a seamless communication and data sharing within the distributed research units.

EPTRI arises from the need to find answers to the serious lack of medicines for children in European Union and worldwide. Minors represent $20 \%$ of the European population and their care is one of priorities and challenges for Europe. ${ }^{2}$ Nevertheless, children are often excluded from the advantages of scientific discovery and innovation, such as new medicinal products. Neonates, children, and adolescents are routinely given adult-approved drugs as "off-label" prescriptions, whereas researchers have demonstrated that children often do not respond to medications as adults and have different (side) effect profiles. Considering the many biological and physiological changes occurring during human development, they represent a "special" population that is indeed made of different age groups with variable pharmacotoxicological characteristics. For this reason, it is important to develop new medicines and therapies for children and young patients by performing dedicated pediatric research.

One of the main key enabling technologies and expertise within EPTRI is represented by the thematic research platform grouping high-quality annotated biosample collections identified in biobanks dedicated to pediatrics. This integrated platform covers these existing gaps in pediatric research by providing scientific competencies, equipment, and highly skilled and trained personnel, and facilitates access to quality controlled biospecimens suitable for research.

The Belgian national EPTRI JRU involves academic research organizations and hospitals from Flanders, Brussels, and Wallonia and will be coaligned with the Belgian Pediatric Clinical Research Network. ${ }^{1}$ Furthermore, there will be a close collaboration at national level as well as at European level between EPTRI and other research infrastructures including Biobanking and BioMolecular resources Research Infrastructure (BBMRI) in the field of pediatric biobanking, ethical, social, and legal issues, ELIXIR (a distributed infrastructure for life-science information) in the field of pediatric data interoperability, the Innovative Medicines Initiative (IMI) Conception project on pharmacotherapy and safety (maternal, fetal, and infant) during pregnancy and lactation, and Conect4children (Collaborative Network for European clinical trials for children).
In Belgium, the national EPTRI JRU will also develop methodologies and quality standards for pediatric biobanking and collect pediatric biospecimens for research. ${ }^{1}$ The pediatricfocused research activities in Belgium will benefit from the development of a pediatric research infrastructure in terms of increased international visibility of the research community, access to industry partners to provide services and start of research collaborations, as well as adaptation of existing infrastructures to include pediatric needs. This unique research framework, including universities and clinical centers of excellence declaring their availability to provide technologies, services, and pediatric expertise, will allow facilitating the translation of the acquired new knowledge and scientific innovation into pediatric clinical study phases and medical use. Sharing understanding of patients' needs and concerted efforts in pediatric research will further enhance the health of children.

\section{Disclaimer}

Prof. Dr. Elke Smits is a member of the European Paediatric Translational Research Infrastructure (ID-EPTRI) Project. ID-EPTRI has received funding from the European Union's Horizon 2020 program under grant agreement No. 777554.

\section{References}

1. De Taeye L, Allegaert K, Smits A, et al. EPTRI Belgian Joint Research Unit: harmonisation and concertation of paediatric research in Belgium to ensure better and safer health care for children. EPTRI Open Meeting, April 2-3, 2020. Available at: https://eptri.eu/wp-content/uploads/2020/ 04/Leen-De-Taeye_-Harmonisation-and-concertation-of-pae diatric-research-in-Belgium-to-ensure-better-and-safer-health care-for-children.pdf; https://www.youtube.com/watch?v= pcqaYBqb9Dk (accessed July 2, 2020).

2. EPTRI Virtual Open meeting, April 2-3, 2020. Available at: https://eptri.eu/eptri-open-meeting/eptri-open-meeting-2/, https://eptri.eu/eptri-open-meeting/poster-presentations/

Address correspondence to:
Elke Smits, PhD
Liaison Officer
On behalf of EPTRI Belgian JRU
Clinical Research Center
University of Antwerp/Antwerp University Hospital
Wilrijkstraat 10
B-2650 Edegem
Belgium

E-mail: elke.smits@uza.be

\section{Expert Response: Engela H. Conradie of the Centre for Human Metabolomics Biobank, North-West University, South Africa}

\footnotetext{
The Centre for Human Metabolomics (CHM) Biobank was established in the beginning of 2019 and is the first congenital rare disease biobank in South Africa and Africa. Congenital disorders are a very important cause of spontaneous abortions, stillbirths, infant and childhood deaths, as well as chronic illness and disability. Congenital
} 
anomalies are furthermore most prominent as a cause of death in settings where overall mortality rates are lower, for example, Europe. Considerable uncertainties remain as to the number of infant and children $<5$ years mortality that are attributed to congenital disorders. ${ }^{1}$

Approximately $50 \%$ of congenital anomalies cannot be linked to a specific cause or risk factor. Furthermore, it is estimated that $94 \%$ of the severe cases of congenital anomalies occur in low- and middle-income countries, including South Africa. According to the WHO 2000-2015 child causes of death, $\sim 11.3 \%$ of neonatal deaths within 4 weeks of birth for the period 2015 could be assigned to congenital anomalies. ${ }^{2}$

Most rare diseases often place a large burden on the patient concerning quality of life and have huge financial implications to the families affected. ${ }^{3}$ The study of rare diseases have proven beneficial for common diseases as well and a greater understanding of human physiology, biological pathways, and human pathology. ${ }^{3,4}$ Garcia et al. have found that rare disease biobanks generated far more publications and had quantifiable more research activity than standalone registries and regular biobanks. ${ }^{3}$

The CHM Biobank's resource stands to benefit the wider South African and global community through the advancement of medical and scientific knowledge, possible treatments, and technological advances through studies that are aligned with the research purpose of the CHM Biobank. The genomic variation of the African population, as well as "treatment-naive" patients, provides added global research value to the biobank. The CHM Biobank is open to national and international collaboration to maximize the utilization of the resource and to optimize research outputs and data sharing. The main criteria for collaboration will be knowledge transfer and capacity building of South African researchers.

\section{References}

1. World Health Assembly (WHA). Resolution 63.17. Birth Defects. Geneva. 2010. Available at: http://apps.who.int/gb/ ebwha/pdf_files/WHA63/A63_R17-en.pdf (last accessed on November 2, 2019).

2. World Health Organisation (WHO). Congenital Anomalies. Fact Sheet. 2016. Available at: https://www.who.int/en/ news-room/fact-sheets/detail/congenital-anomalies (last accessed on November 2, 2019).

3. Garcia M, Downs J, Russel A, et al. Impact of biobanks on research outcomes in rare diseases: A systematic review. Orphanet J Rare Dis 2018;13:202.

4. Hall AK, Sireau NT. Findacure-The fundamental diseases partnership. Orphanet J Rare Dis 2014;9(Suppl 1):O23.

Address correspondence to: Engela H. Conradie, PhD, PrChemSA North-West University, Potchefstroom Campus Centre for Human Metabolomics Building F3, 11 Hoffmann Street Potchefstroom 2531 South Africa

E-mail: elne.conradie@nwu.ac.za 